\title{
A lineage-specific epigenetic memory of inflammation potentiates Kras- driven pancreatic tumorigenesis
}

\author{
David J. Falvo ${ }^{1,2}$, Adrien Grimont ${ }^{1,2}$, Paul Zumbo ${ }^{3,4}$, Julie L. Yang ${ }^{5}$, Alexa Osterhoudt',2, Sarah L. Breves ${ }^{1,2}$, Grace \\ Pan $^{1,2}$, Andre F. Rendeiro ${ }^{3,6}$, John E. Wilkinson ${ }^{7}$, Friederike Dündar ${ }^{3,4}$, Olivier Elemento ${ }^{3,6}$, Rhonda K. Yantiss ${ }^{8}$, Doron \\ Betel $^{3,4,9}$, Richard Koche ${ }^{5}$, and Rohit Chandwani ${ }^{1,2,10, *}$
}

\begin{abstract}
Tissue homeostasis depends on responses to environmental insults to restore cellular phenotype, microenvironment composition, and tissue architecture. Inflammation is essential to the disruption of homeostasis in epithelial tumorigenesis, but how a temporally remote inflammatory episode impacts tumor development is unknown. Herein we employ lineage-traced mouse models to unveil the presence of an epigenetic memory of inflammation. We observe that despite histologic resolution of pancreatitis, acinar cells fail to return to the same molecular baseline. In vivo, the memory is associated with diminished metaplasia in response to a second inflammatory insult but increased tumorigenesis when instead subjected to an oncogenic Kras mutation. We find that memory is a cell-intrinsic property, primarily encoded in chromatin, that features persistent derepression of metaplastic genes and is recalled with oncogenic stress. Together, our findings define a capacity for an environmental insult to potentiate future tumor initiation, broadening the relationship between inflammation and cancer.
\end{abstract}

\section{SIGNIFICANCE}

Inflammation is a key characteristic of epithelial cancers. Here, we demonstrate that resolution of pancreatitis in vivo is accompanied by memory, featuring persistent epigenetic and transcriptional alterations that are recalled to increase the susceptibility to oncogenic stress later in life.

\section{INTRODUCTION}

Biological systems operate within fluctuating environments, and, therefore, are inherently tasked with accurately responding in real-time to a myriad of signals. Preservation of tissue homeostasis might also require adaptation to potential future contexts as well. Recent evidence suggests that 'memories' of transient stimuli can be encoded and retained in the epigenome of cells even following resolution of the initial stimulus. ${ }^{1-3}$ While this phenomenon is welldescribed in the nervous system and immune system, whether memory is a feature of other differentiated somatic cell types is largely unknown.

In the pancreas, inflammation, which is known to play varying roles in both tumor initiation and progression, results in a transient cell-fate transition known as acinar-to-ductal metaplasia (ADM). ADM precedes the development of pancreatic intraepithelial neoplasia (PanIN) and subsequent pancreatic ductal adenocarcinoma (PDAC). The earliest preclinical models employing oncogenic Kras activated by embryonic Cre drivers demonstrated sufficiency of mutant Kras to unveil PanIN and PDAC. ${ }^{4}$ However, in the adult mouse, inflammation, typically experimentally elicited by the cholecystokinin analog caerulein, is necessary to give rise to neoplastic lesions that are most effectively derived from acinar cells. ${ }^{5-8}$ Recent data highlight the capacity of inflammation to destabilize pancreatic epithelial cell identity $^{9-10}$ to promote tumor initiation via acquisition of progenitor features ${ }^{11-12}$ and/or outgrowth of specific critical niche populations. ${ }^{13}$ These models demonstrating cooperativity between oncogenic stress and inflammation largely utilize contemporaneous insults, such that the lasting impact of inflammation on pancreatic tissue homeostasis has been mostly unexplored.

In the patient setting, it has been observed that chronic pancreatitis is a well-established risk factor for pancreatic cancer. Indeed, germline mutations in the PRSS1 gene causing hereditary pancreatitis drive a substantial increase in the risk of PDAC. Surprisingly, a single episode of selflimited acute pancreatitis is suggested to confer an increased risk of developing subsequent PDAC up to 10 years after the episode. ${ }^{14}$ Here, we offer one potential explanation for this phenomenon. By leveraging lineage tracing of pancreatic acinar cells, we define the long-term ramifications of a transient inflammatory insult on pancreatic tissue homeostasis and tumorigenesis. We find that inflammation has a prolonged and durable impact on acinar cell identity, and this alters the responses to a secondary inflammatory insult and to delayed activation of mutant Kras. Our data thus highlight the ability for remote inflammation to become encoded as a lasting acinar cell-specific epigenetic memory of the prior insult and to drive tumor development in vivo.

\section{RESULTS}

A transient inflammatory episode induces persistent molecular alterations despite histologic resolution

We first asked whether a transient inflammatory episode persistently alters the transcriptional profile and chromatin accessibility landscape of pancreatic acinar cells. We used Mist1-Cre ERT2; LSL-tdTomato (MT) mice to conditionally restrict tdTomato expression to the acinar compartment after tamoxifen treatment. ${ }^{7}$ After tdTomato activation, a transient inflammatory insult was induced in MT mice via intraperitoneal administration of caerulein for 3 weeks ${ }^{15}$

\footnotetext{
${ }^{1}$ Department of Surgery, Weill Cornell Medicine, New York, NY; ${ }^{2}$ Sandra and Edward Meyer Cancer Center, Weill Cornell Medicine, New York, NY; ${ }^{3}$ Institute for Computational Biomedicine, Department of Physiology, Biophysics, and Systems Biology, Weill Cornell Medicine, New York, NY; ${ }^{4}$ Applied Bioinformatics Core, Weill Cornell Medicine, New York, NY; ${ }^{5}$ Center for Epigenetics Research, Memorial Sloan Kettering Cancer Center, New York, NY; ${ }^{6}$ Caryl and Israel Englander Institute for Precision Medicine, Weill Cornell Medicine, New York, NY; ${ }^{7}$ Department of Pathology, University of Michigan, Ann Arbor, MI; ${ }^{8}$ Department of Pathology, Weill Cornell Medicine, New York, NY; ${ }^{9}$ Division of Hematology and Oncology, Department of Medicine, Weill Cornell Medicine, New York, NY; ${ }^{10}$ Department of Cell and Developmental Biology, Weill Cornell Medicine, New York, NY; ${ }^{*}$ Corresponding author: Rohit Chandwani, MD, PhD, roc9045@med.cornell.edu
} 
A

Mist1-Cre ${ }^{\text {ERT2. }}$ CAGs-LSL-TdTomato
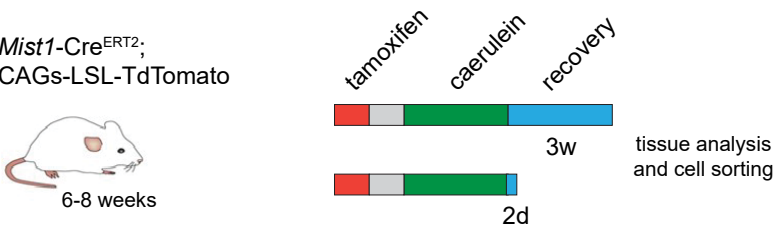

B

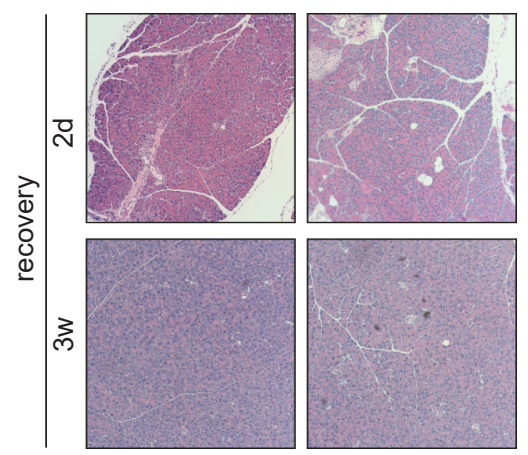

C
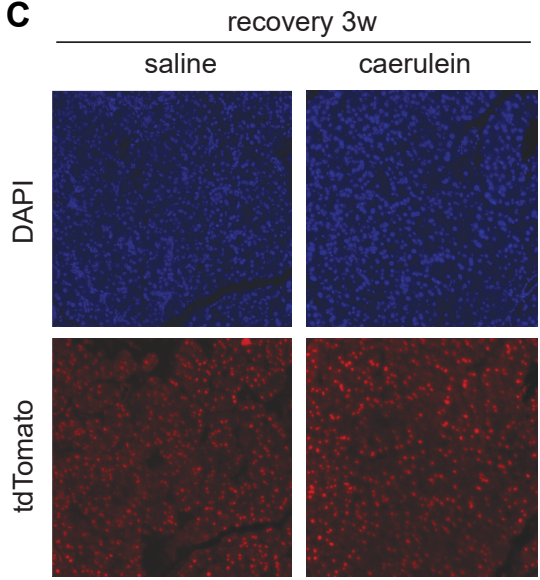

$\frac{\pi}{\widetilde{\jmath}}$
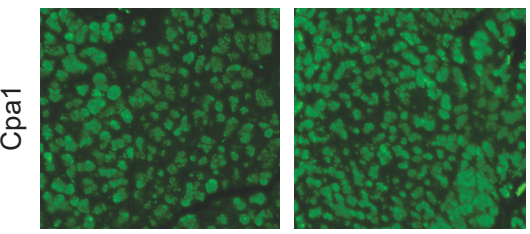

$\frac{\sigma}{\bar{x}}$
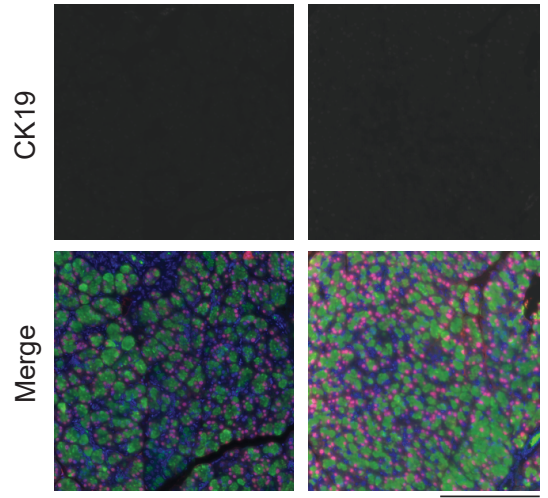

$200 \mu \mathrm{m}$

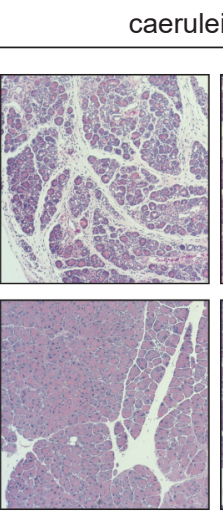

E

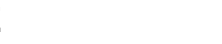

$\mathbf{F}$
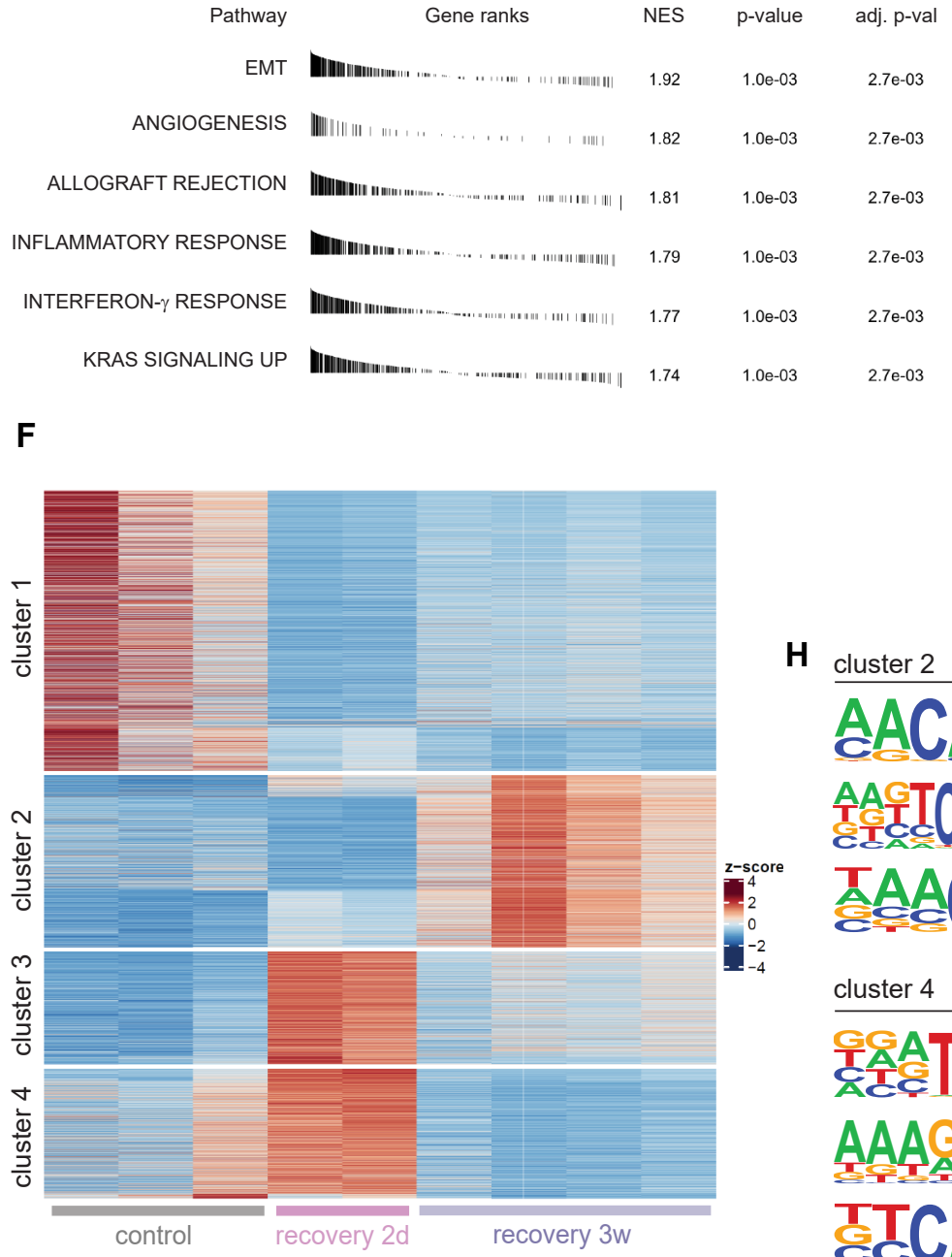

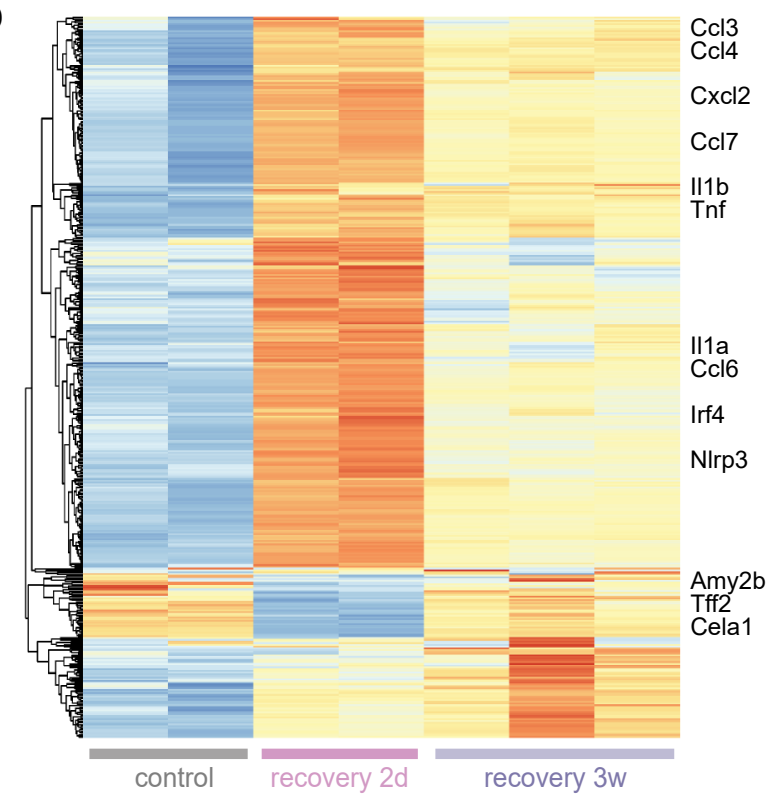

G

RNA-seq

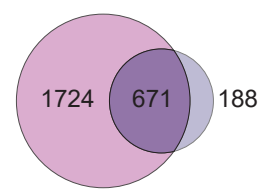

ATAC-seq

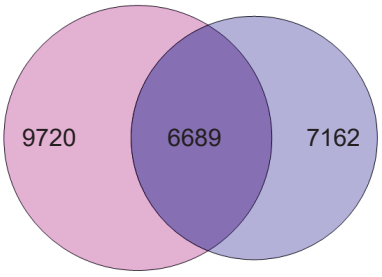

$\square$ recovery 2d (vs. control)

$\square$ recovery $3 \mathrm{w}$ (vs. control)

H cluster 2 cluster 2

GO: CC proteinaceous extracellular matrix

adj p-value

GO: CC extracellular matrix

GO: MF

GO: CC

GO: MF metallopeptidase activity

extracellular space

metalloendopeptidase activity
$1.88 \mathrm{E}-38$

2.14E-32

1.20E-24

$3.46 \mathrm{E}-24$

3.60E-24 cluster 4

GO: BP

GO: BP

GO: CC

GO: BP

GO: CC
AAC AACTCCTTACGCAAT Eer2 10609

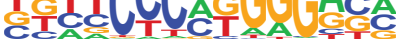

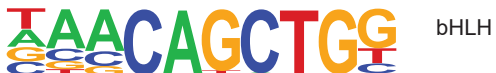

1e-60

cluster 4

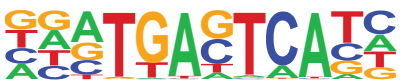

AP-1

$1 \mathrm{e}-134$ AAAGACOAAG ETS 1e-54 ECCAAGGTCA 
followed by recovery intervals of 2 days and 3 weeks (Figure 1A). We observed that after 2 days of recovery, pancreatic histology exhibited pronounced metaplasia and tissue damage (Figure 1B). At 3 weeks of recovery, the pancreas was devoid of ADM and inflammatory infiltrates, with absence of the ductal marker CK19 from acinar cells, indicating redifferentiation of the acinar compartment (Figures 1C).

To assess the spectrum of molecular changes induced during and after inflammation, pancreata were harvested from both MT mice treated with caerulein, at 2 days and 3 weeks after the insult. tdTomato(+) acinar cells were isolated via fluorescence-activated cell sorting (FACS) for downstream profiling of transcriptional and chromatin accessibility changes using RNA-seq and ATAC-seq, respectively. At 2 days after caerulein (recovery $2 d$ ), we observed an expected downregulation of acinar-specific genes (e.g. Amy2b, Tff2, Cela1), with strong upregulation of pro-inflammatory genes (e.g. $\mathrm{Ccl} 3, \mathrm{Ccl} 4, \mathrm{Cxcl} 2, \mathrm{Il1b}, \mathrm{Tnf}$ ). Surprisingly, we found that after 3 weeks (recovery $3 w$ ), acinar cells display a mixed transcriptional state, characterized by persistent expression of metaplasiaassociated genes despite normal histology (Figure 1D). Using Gene Set Enrichment Analysis (GSEA), we observed an enrichment of transcripts involved in epithelial-tomesenchymal transition (EMT), angiogenesis, inflammation, and related to Kras signaling at 3 weeks of recovery -- albeit to a lesser extent than at 2 days (Figure 1E; Figure S1A).

Next, we evaluated the changes in chromatin accessibility at the peak of inflammation and after resolution of pancreatitis. Principal Component Analysis (PCA) on the ATAC-seq data from FACS-sorted acinar cells collected from all three conditions showed separation between recovery $2 \mathrm{~d}$ and recovery $3 d$ mice; consistent with the RNA-seq data, chromatin accessibility remained altered from baseline after 3 weeks of recovery (Figure S1B). Inflammation-exposed tdTomato(+) acinar cells collected after 2 days of recovery exhibited dramatically reduced accessibility at genomic regions enriched in control acinar cells (Figure 1F; Cluster 1), suggesting a loss of acinar cell identity. Additionally, we observed profound gains in accessibility at intergenic and intronic regions unveiled during peak inflammation (Figure 1F; Clusters 3 and 4). Interestingly, we found that there were novel gains of accessibility at 3 weeks of recovery (Cluster 2) and dramatic losses of accessibility in Cluster 4, highlighting ongoing molecular changes in the acinar compartment well after the inflammatory insult.

To understand the scope of these molecular alterations, we compared the degree of overlap between 2 days and 3 weeks of recovery for both the transcriptional and chromatin accessibility changes observed. Whereas the majority of persistent transcriptional changes at 3 weeks were a subset of the 'recovery $2 d$ ' differentially expressed genes (DEGs), the differentially-accessible regions (DARs) specific to 3 weeks of recovery were greater in number and more distinct (Figure 1G). This suggests that chromatin -- to a greater extent than the transcriptome - remains dynamic up to 3 weeks after a transient insult.

Next, we performed motif analysis on the clustered DARs, identifying a number of acinar-specific factors (e.g. Ptfla, Bhlha15) in Cluster 2, while motifs for factors involved in pancreatitis were found in Cluster 4 (e.g. AP-1, Nr5a2) (Figure $1 \mathrm{H}$; Figure S1C). Broadly, 3 weeks of recovery restored accessibility of regions enriched in motifs for lineagespecifying TFs (Nr5a2, Bhlha15, Nkx6.1) but showed persistent depression of AP-1 motifs (Figure S1D). Gene Ontology (GO) enrichment analysis on the clustered DARs identified a number of processes associated with the extracellular matrix (ECM) in Cluster 2, suggesting that there are ongoing changes in tissue architecture at 3 weeks of recovery, perhaps in keeping with the loss of cellularity seen by $H \& E$ (Figure 1B). In Cluster 4, where there is rapid resolution of transient increases in accessibility, we found GO terms associated with processes characteristic of pancreatitis (e.g. immune system process, exocytosis). These findings demonstrate that inflammation-resolved acinar cells retain expression of genes upregulated during pancreatitis. However, these transcriptional changes are relatively small compared to the chromatin changes, where acinar cells retain and even accumulate clear gains in accessibility at sites normally absent in control acinar cells, while also failing to re-establish baseline accessibility at acinar-specific locations.

\section{Memory of inflammation manifests as an incomplete cell fate decision \\ Because we observed emergent acinar cell alterations at 3 weeks following pancreatitis, we asked if these molecular dynamics resolve over time. We therefore treated mice as previously described, but extended the recovery window to include 6,12 , and 18-week recovery timepoints. At 12 and 18 weeks of recovery, we saw continued evidence of normal tissue architecture, and no persistent changes to the abundance of the ADM/ductal markers Sox9 and CK19 (Figure S2A-B) nor the acinar marker Cpa1 (Figure S2B). We evaluated the persistence of phospho-Erk (Indicating MAPK pathway activation), or the progenitor markers KIf5 and Nestin; while these were robustly induced at 2 days, there}

\footnotetext{
Figure 1. A transient inflammatory episode induces persistent molecular alterations despite histologic resolution. A, Schematic representation of lineagetraced mouse model with treatment regimen. B, Hematoxylin and eosin staining of mouse pancreas sections collected 2 days and 3 weeks after caeruleininduced pancreatitis. C, Immunofluorescence for DAPI, tdTomato, Carboxypeptidase A1 (Cpa1), and Cytokeratin 19 (CK19) staining of pancreas sections collected 3 weeks after caerulein-induced pancreatitis. Images are representative of N = 3 mice per condition. D, Heatmap of RNA-seq data generated with the top 100 differentially expressed genes (thresholds: $\log _{2}$ (fold change) > abs(2); p-value < 0.01) when comparing inflammation-naïve tdTomato(+) acinar cells (control) and peak inflammation tdTomato(+) acinar cells (recovery 2d); supervised clustering according to indicated condition. Each column is a biological replicate mouse. E, Gene Set Enrichment Analysis (GSEA) of RNA-seq data between control and recovery $2 \mathrm{~d}$ condition, top six results are shown. F, Heatmap of differentially accessible regions (DARs) from bulk ATAC-seq data, generated with k-means cluster analysis across the indicated conditions; color reflects z-scores of chromatin accessibility where each row is a specific chromatin peak. Each column is a biological replicate mouse. G, Venn diagrams illustrating the degree of overlap between 2 days of recovery versus control and 3 weeks of recovery versus control from a transcriptional (top) and chromatin accessibility (bottom) standpoint; log2FC $>1$; FDR $<0.05$. H, HOMER analysis depicting top three motif enrichments in Cluster 2 and Cluster 4 and associated adjusted p-values. I, GO term enrichment for genes associated with differentially accessible sites in Cluster 2 and Cluster 4 . Top five results are shown along with adjusted $\mathrm{p}$-values. $\mathrm{CC}=$ cellar component; $\mathrm{BP}=$ biological process; $\mathrm{MF}=$ molecular function .
} 
bioRxiv preprint doi: https://doi.org/10.1101/2021.11.01.466807; this version posted November 4, 2021. The copyright holder for this preprint (which was not certified by peer review) is the author/funder. All rights reserved. No reuse allowed without permission.

A

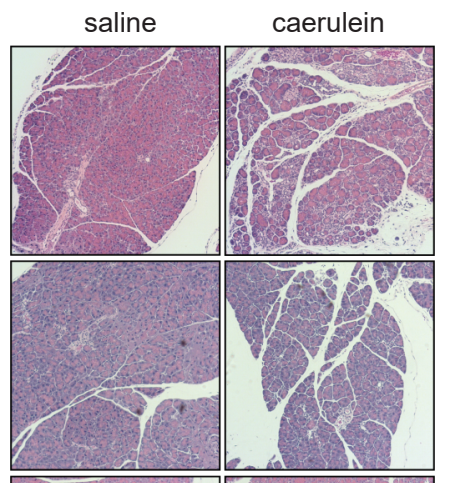

$12 w$

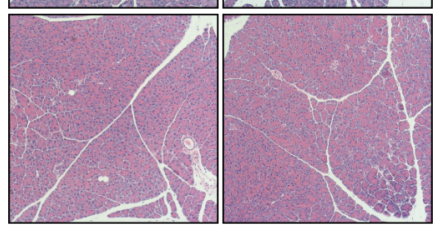

$18 w$

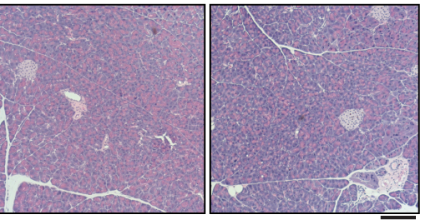

$100 \mu \mathrm{m}$

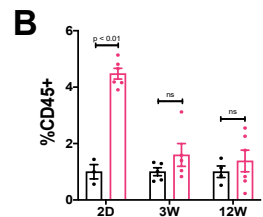

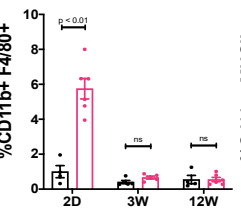

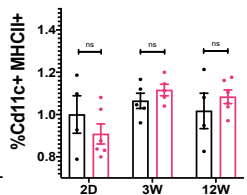

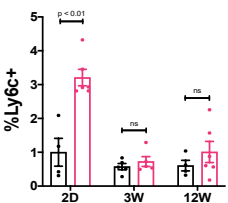

蒂

C
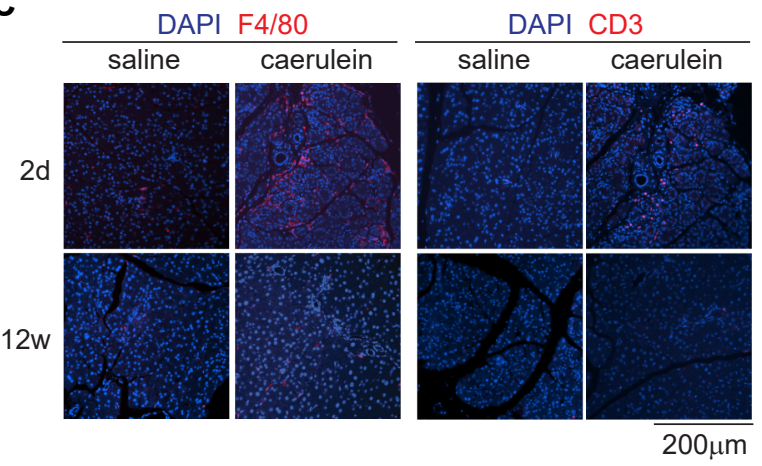

D
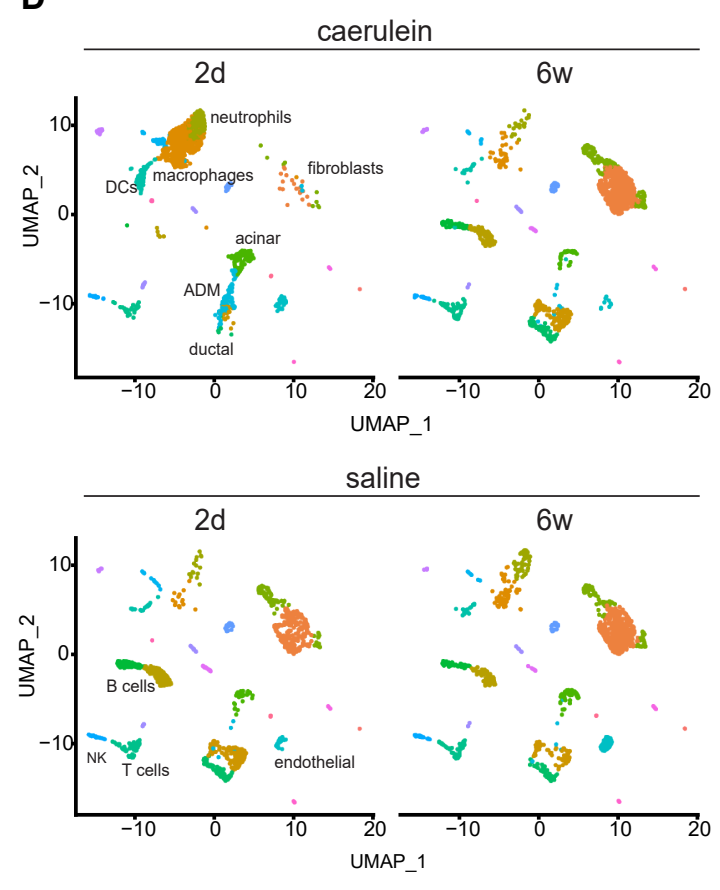

E

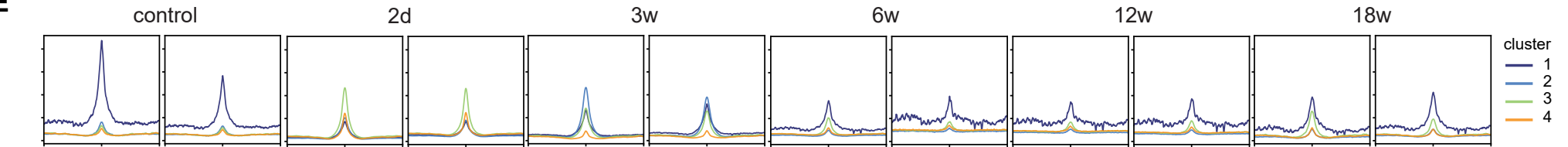

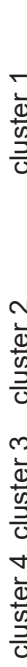
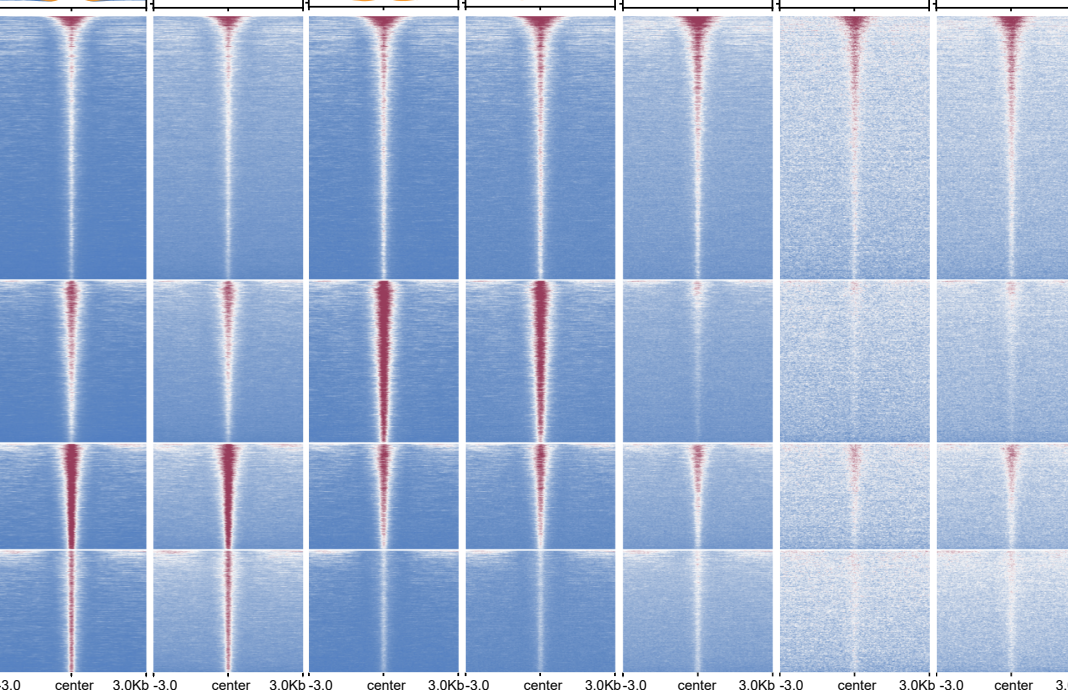

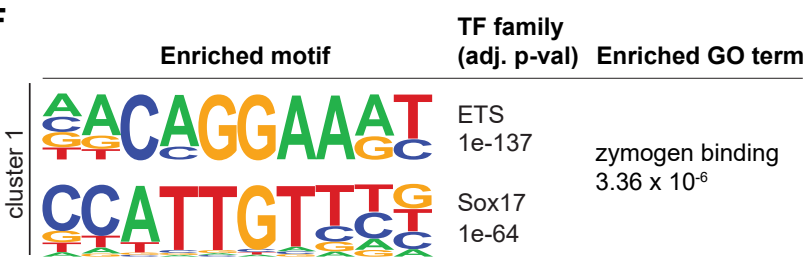

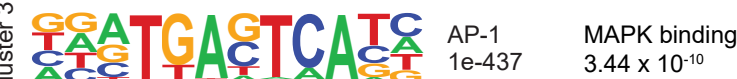

H

Pathway Gene ranks NES p-val adj.p

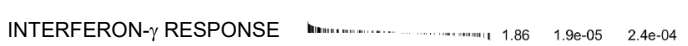

INTERFERON- $\alpha$ RESPONSE

E2F TARGETS

OXIDATIVE PHOSPHORYLATION
G

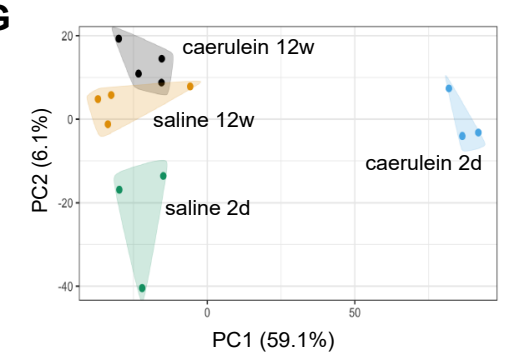

ADM transcripts

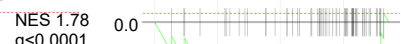

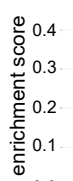

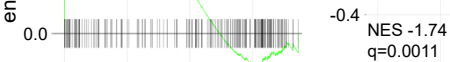

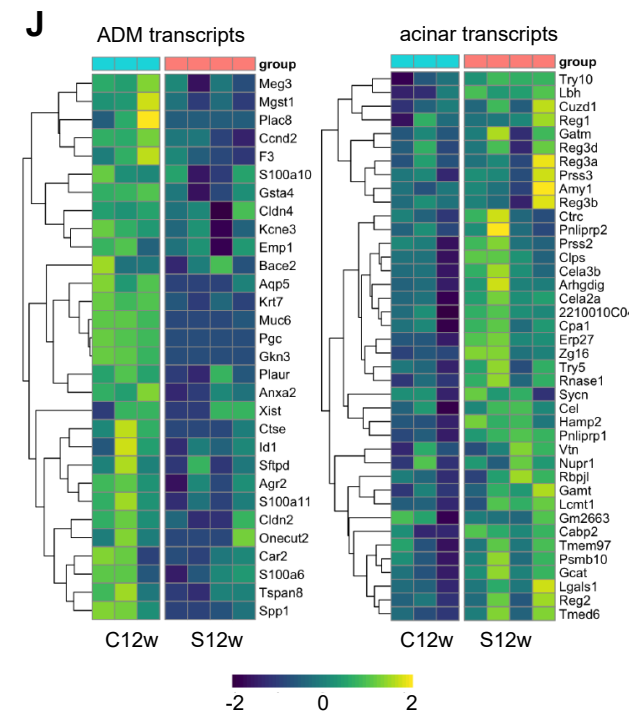


bioRxiv preprint doi: https://doi.org/10.1101/2021.11.01.466807; this version posted November 4, 2021. The copyright holder for this preprint (which was not certified by peer review) is the author/funder. All rights reserved. No reuse allowed without permission.

Falvo et al, Epigenetic memory of inflammation in acinar cells potentiates Kras-driven pancreatic tumorigenesis

was no lasting expression at 12 weeks (Figure S2C). We thus concluded that 12 weeks of recovery was indeed sufficient to restore typical markers of pancreatic epithelial cell homeostasis.

We then asked if there was a persistent microenvironment change that accompanied the recovery from pancreatitis. Profiling of the immune compartment (via flow cytometry) showed robust infiltrates of macrophages and monocytes at 2 days that returned to baseline levels with 3 and 12 weeks of recovery (Figure 2B-C). Single-cell RNA-sequencing of isolated pancreas from mice following recovery from acute caerulein showed neither a persistent immune infiltrate at 6 weeks, nor a shift in transcriptional state among immune cells, fibroblasts, endothelial cells. Importantly, we did detect the abundant infiltration of myeloid cells and emergence of ADM cells at 2 days, but no persistent alteration over time (Figure 2D). In these data, we observed that ADM cells manifest as a mixed transcriptional state, characterized by the expression of genes enriched in both acinar and ductal cells (Figures S2D and E). However, scRNA-seq did not reveal persistence of a metaplastic population with recovery from pancreatitis.

Next, we determined if chromatin alterations in acinar cells persist despite our data suggesting a restoration of tissue architecture, gene expression, and microenvironment composition with time. To this end, we obtained sorted tdTomato(+) acinar cells from the 6,12 , and 18 -week recovery timepoints for subsequent downstream profiling of chromatin accessibility. Unexpectedly, we found that even after 18-weeks of recovery, acinar cells retained a chromatin signature of prior inflammation (Figure 2E; Cluster 3). Motif enrichment of these gained memory regions showed an abundance of AP-1 motifs, with a top gene ontology corresponding to MAPK pathway (Figure 2F). Conversely, acinar cell identity regions in Cluster 1 failed to completely restore chromatin accessibility present in control acinar cells, even after 12 and 18 weeks of recovery. We found that the sites in Cluster 1 were enriched for ETS factor and Sox17 motifs, known to be involved in pancreas development, and identified GO terms associated with processes important for acinar cell function (i.e. zymogen binding). Interestingly, regions enriched in Cluster 2 that initially increased accessibility up to 3 weeks of recovery were markedly lost at 6 weeks of recovery and beyond, suggesting that the full range of chromatin dynamics are more adequately revealed with prolonged recovery (Figure 2E). Together, these data highlight a prolonged cell-intrinsic effect of prior inflammation to chromatin.

To determine if persistent transcriptional changes accompanied the chromatin dynamics, we performed bulk RNA-seq on sorted tdTomato(+) acinar cells. We found little to no shift in clustering of caerulein +12 week recovery (caerulein 12w; C12w) samples versus saline +12 week recovery (saline 12w; S12w) samples (Figure 2G). No significant individual gene expression changes were found at this timepoint (Figure S2F), but at the pathway level, there were increases in both inflammatory and metaplasia-specific (ADM) transcripts $^{16}$ in caerulein $12 \mathrm{w}$ samples; conversely, acinar transcripts ${ }^{16}$ were enriched in inflammation-naïve acinar cells (Figures $2 \mathrm{H}-\mathrm{I}$ ). Further, leading-edge ADM genes increased in expression in $\mathrm{C} 12 \mathrm{w}$ contained nearby regulatory elements with persistently increased accessibility (i.e. cluster 3-type), while acinar gene-related enhancers showed cluster 1 dynamics (Figure S2G). These results suggest that prior injury generate a memory of inflammation manifesting as an incomplete cell fate decision, where both chromatin alterations and transcriptional states show residual evidence of the remote metaplasia event.

\section{Prior inflammation alters the capacity for subsequent metaplasia}

Given these findings, we asked whether the memory of inflammation impacts acinar cell responses to future stimuli. We first tested if memory predisposes acinar cells to undergo promiscuous metaplasia. To test this, we treated wild-type C57BL/6 mice to a primary stimulus of either saline or caerulein followed by a recovery period and then secondary treatment with caerulein. With a recovery period of 3 weeks, we observed that secondary caerulein elicited more robust ADM in mice previously treated with caerulein (Figure S3A-B); however, we detected residual ADM foci in mice initially treated with caerulein and followed by saline, underscoring our findings that tissue homeostasis is not fully restored at 3 weeks.

We thus focused on allowing for 12 weeks of recovery between primary and secondary insults (Figure 3A). Surprisingly, we found pancreata exposed to prior inflammation are refractory to subsequent pancreatitis, with increased ADM in inflammation-naive mice compared to inflammation-resolved mice in response to secondary administration of caerulein (Figures 3B-C). Co-staining of Cpa1 and CK19 was observed in inflammation-resolved

Figure 2. Memory of inflammation manifests as an incomplete cell fate decision. A, Hematoxylin and eosin staining of saline or caerulein treated mouse pancreas sections collected after 2 days, 6,12 , and 18 weeks of recovery. B, Flow cytometric quantification of CD45(+), CD11b(+) F4/80(+), CD11C(+) MHCll(+), Ly6c(+), CD3(+), CD19(+) immune cell populations showed in percentage of isolated cells from pancreata of inflammation-naïve (saline) and inflammationexposed (caerulein) mice collected after 2 days, 3 weeks, and 12 weeks of recovery. Student's t-test was performed between conditions. N=3-5 mice per condition. C, Immunofluorescence for F4/80, CD3 and DAPI of pancreas sections collected 2 days and 12 weeks after caerulein-induced pancreatitis. D, UMAP projection of single-cell RNA-seq data generated with cells isolated from pancreata collected 2 days and 6 weeks after saline or caerulein treatment. $\mathbf{E}$, Tornado plots visualizing the chromatin accessibility upstream and downstream of a 6 -kilobase window (i.e. $-3 \mathrm{~kb}$ to $+3 \mathrm{~kb}$ ) at different locations throughout the genome (the clusters and genomic locations populating each cluster are identical to the sites in Figure 1F). Red indicates maximal signal and blue signifies absent signal. Each row corresponds to a genomic location, and each column represents a single biological replicate mouse treated according to the conditions indicated. Two mice per condition are shown, representative of $\mathrm{N}=2-5$ mice per condition. $\mathbf{F}$, HOMER analysis depicting motif enrichment in Cluster 1 and Cluster 3 (with adjusted p-values), as well as the top GO term (with associated adjusted p-value) associated with sites enriched Cluster 1 and Cluster 3. G, Principal Component Analysis (PCA) plot of bulk RNA-seq data generated from tdTomato(+) acinar cells collected 2 days and 12 weeks after either saline or caerulein treatment. H, GSEA of RNA-seq data derived from inflammation-naïve tdTomato(+) acinar cells versus inflammation-exposed tdTomato $(+)$ acinar cells (12 weeks of recovery). Top 4 most up- or down-regulated pathways are shown. I, GSEA enrichment plot of RNA-seq data derived from caerulein $12 w$ (caerulein $+12 w$ recovery) vs. saline $12 w$ (saline $+12 w$ recovery) samples for the indicated ADM and acinar gene sets (obtained from ref. 16) J, Heatmap of RNA-seq data delineating the leading edge ADM and acinar transcripts in C12w and S12w samples; each column represents a single biological replicate mouse. 
bioRxiv preprint doi: https://doi.org/10.1101/2021.11.01.466807; this version posted November 4, 2021. The copyright holder for this preprint (which was not certified by peer review) is the author/funder. All rights reserved. No reuse allowed without permission.

A

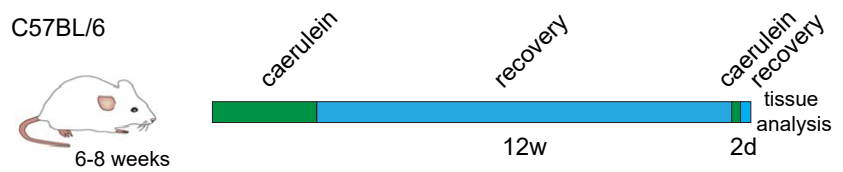

B
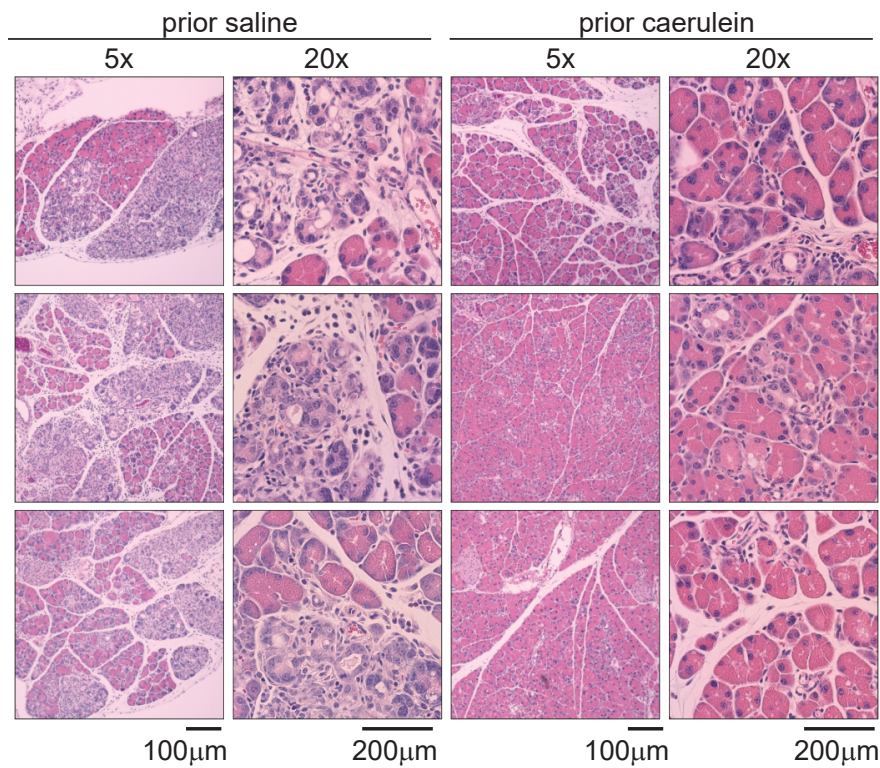

D
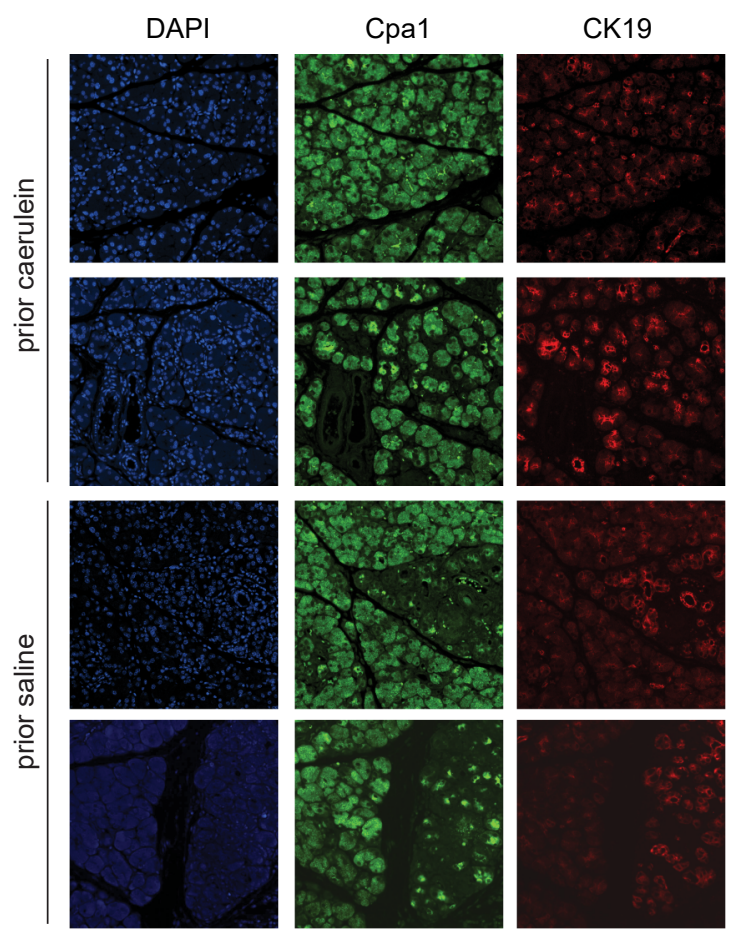

C

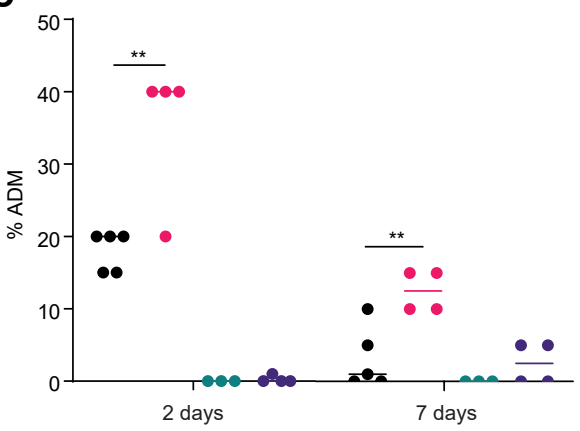

- prior caerulein $\mid 2^{\circ}$ caerulein

- prior saline $\mid 2^{\circ}$ caerulein

- prior saline $\mid 2^{\circ}$ saline

- prior caerulein $\mid 2^{\circ}$ saline
E

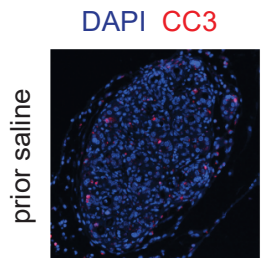

$\mathbf{F}$

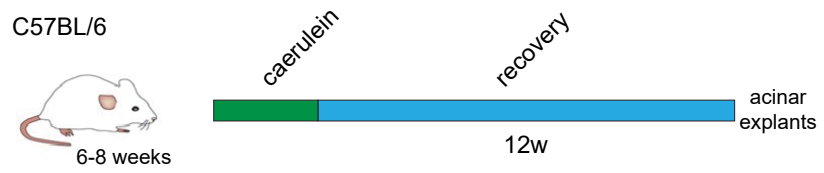

G
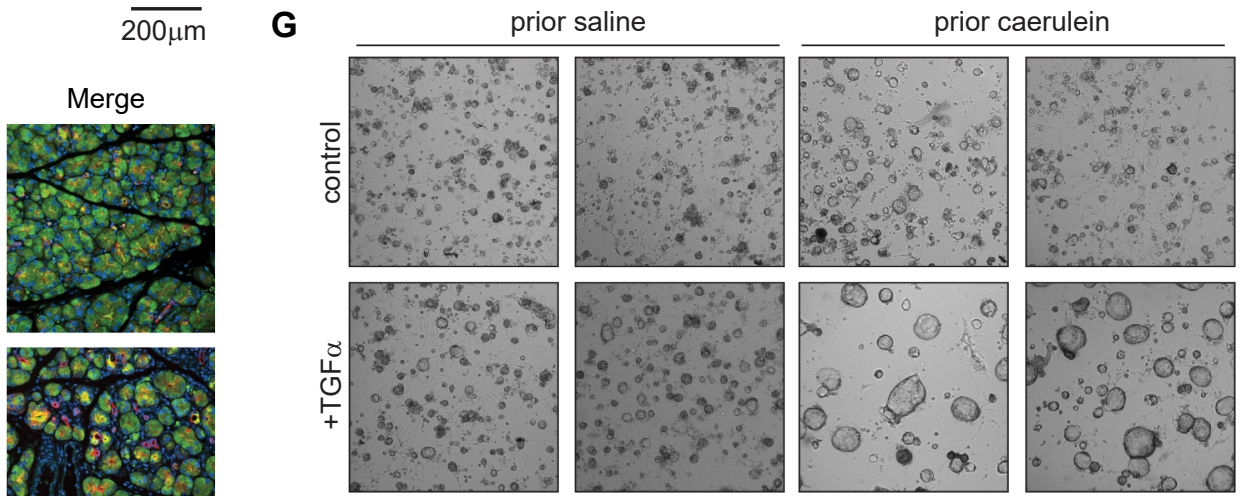

H

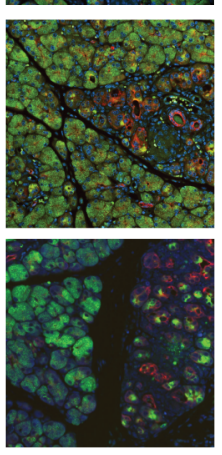

$200 \mu \mathrm{m}$

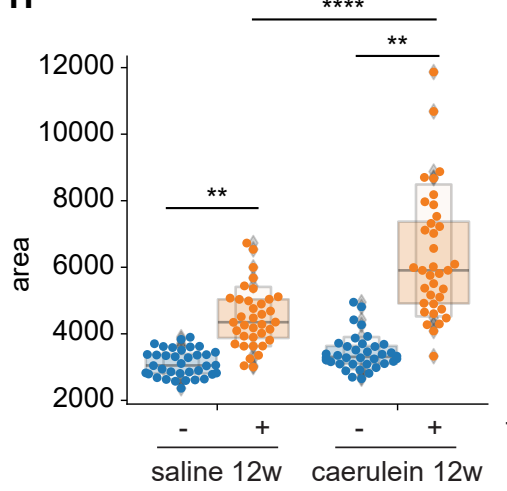

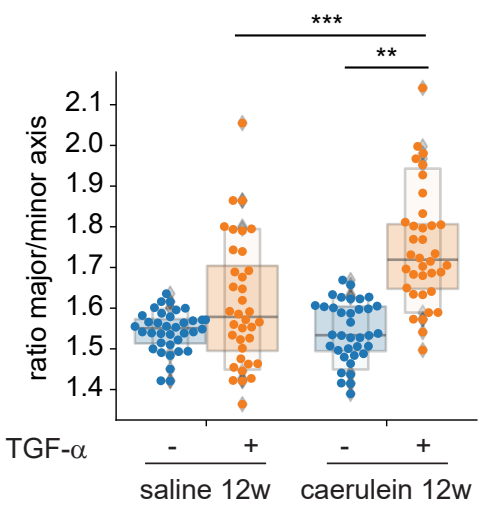

Figure 3. Prior inflammation alters the capacity for subsequent metaplasia. A, Schematic representation of primary pancreatitis and inflammatory rechallenge treatment regimen in wild-type mice. B, Hematoxylin and eosin staining of inflammation-naïve $(\mathrm{N}=4)$ and inflammation-resolved $(\mathrm{N}=5)$ mouse pancreas sections collected 2 days after inflammatory re-challenge. $\mathbf{C}$, Quantification of the ADM area (in percentage) of pancreas sections collected from inflammation-naïve and inflammation-resolved mice re-challenged with either saline or caerulein ( $\mathrm{n}=3-5$ per condition). Student's t-test was performed to compare conditions. D, Immunofluorescence for Cpa1 and CK19 and DAPI staining of inflammation-naïve and inflammation-resolved mouse pancreas sections collected 2 days after inflammatory re-challenge. Representative images shown are from a total of $\mathrm{N}=2-5$ mice per condition. $\mathbf{E}$, Representative immunofluorescence for cleaved caspase 3 (CC3) and DAPI staining of inflammation-naïve and inflammation-resolved mouse pancreas sections collected 2 days after inflammatory re-challenge. Representative images shown are from a total of $\mathrm{N}=2-5$ mice per condition. $\mathbf{F}$, Schematic representation of primary pancreatitis followed by prolonged recovery and acinar explant generation. $\mathbf{G}$, Representative images of acinar explants generated from inflammation-naïve and inflammation-resolved mice re-challenged in vitro with either vehicle or recombinant human TGFa; $\mathrm{N}=4$ mice per condition. $(\mathbf{H})$ Boxplots of image analysis quantifying the area and major/minor axis ratio for inflammation-naive and inflammation-resolved explants treated with vehicle or recombinant human TGFa. Each point represents an object in one of 3 wells per mouse, $\mathrm{N}=4$ mice per condition. Mann-Whitney was performed to compare conditions. 
tissue re-challenged with caerulein, indicative of early ADM, while Cpa1 was lost in the metaplastic lesions of inflammation naïve tissue re-challenged with caerulein, suggesting more advanced ADM (Figure 3D). In addition, cleaved-caspase 3 (CC3) staining co-localized with advanced metaplastic lesions, was proportional to the degree of metaplasia, and not otherwise differentially enriched depending on the prior insult (Figure 3E). We performed similar in vivo re-challenge experiments adjusting the secondary caerulein insult for re-challenge, again observing more metaplasia in inflammation-naïve pancreata as compared to inflammation-exposed pancreata (Figure S3C-D).

Next, we cultured acinar cells to test if prior inflammation alters the capacity of acinar cells to transdifferentiate in vitro in a cell-autonomous fashion. As before, we exposed wildtype C57BL/6 mice to a primary stimulus of either saline or caerulein followed by a recovery period of 12 weeks, and isolated acinar cells for embedding in Matrigel (Figure 3F). In the absence of TGF $\alpha$, we observed no change in acinar cells from naïve or caerulein-treated mice (Figure 3G, upper panels). Treatment with rhTGF $\alpha$ in vitro, which activates signaling downstream of EGFR, gave rise to ductal cystic structures (Figure 3G, lower panels). Interestingly, inflammation-resolved acinar cells generated substantially larger cystic structures when compared to inflammationnaive acinar cells (Figure $3 \mathrm{H}$ ). Our results highlight that the impact of prior inflammation is to diminish overt metaplastic lesions in vivo, but to potentiate the specific response to EGFR-MAPK signaling pathway activation in vitro.

Prior inflammation lowers the threshold for subsequent Kras-driven tumor initiation

We reasoned that the heightened response to a specific EGFR ligand would imply that prior exposure to inflammation lowers the threshold for malignant transformation. Most studies of adult mutant Kras activation utilize a contemporaneous inflammatory insult; therefore, we first sought to determine if any temporal separation between inflammation and oncogene activation could drive tumorigenesis. To test this, we treated MT and Mist1-Cre ${ }^{\text {ERT2; }}$ LSL-Kras $^{\text {G12D/+; }}$, LSL-tdTomato (MKT) mice with either saline or caerulein as before, followed by recovery, and then activation of Kras ${ }^{\mathrm{G12D}}$ with tamoxifen (Figure 4A; Figure S4A). Importantly, this system provided complete temporal control over endogenous levels of Kras expression and with specific restriction to pancreatic acinar lineage. With 3 weeks of recovery between caerulein and Kras $^{\mathrm{G} 12 \mathrm{D}}$ activation (Figure S4A), we indeed observed substantial PanIN development in mice treated previously with caerulein, as compared to rare lesions in naïve mice (Figures S4B). Extension of the temporal separation to 12 weeks (Figure 4A) showed increased ADM and PanIN in mice previously exposed to caerulein (Figures 4B, 4C [right panel], 4D). Immunofluorescent staining of both inflammation-naïve and inflammation-exposed MKT pancreas sections revealed the loss of Cpa1, as well as the colocalization of CK19, at these PanIN lesions (Figure 4E). We also found that the PanIN lesions were enriched with $\mathrm{Dclk1} 1^{+}$cells, as previously described ${ }^{12}$, but not to different degrees between comparable lesions in the two conditions (Figure 4F).
Next, we evaluated the synergistic limits of the inflammatory memory combined with oncogenic Kras by restricting the duration of $\mathrm{Kras}^{\mathrm{G}}{ }^{12 \mathrm{D}}$ activation to a brief window of 2 days (Figure S4C). Surprisingly, even short-term activation of $\mathrm{Kras}^{\mathrm{G} 12 \mathrm{D}}$ was sufficient to induce neoplastic transformation in inflammation-exposed acinar cells; pancreata of inflammation-naïve mice were devoid of any PanIN, and displayed only sparse early metaplastic lesions (Figure 4C; left panel and Figures S4D-F). These findings indicate differential responses to oncogenic Kras that are driven by a temporally remote prior inflammatory episode.

To understand the molecular underpinnings of this phenotype, we performed RNA-seq on inflammation-naïve and -exposed tdTomato(+) acinar cells subjected to brief Kras $^{\mathrm{G} 12 \mathrm{D}}$ activation. As expected, we found that transcripts associated with PanIN and proliferation were specifically upregulated with prior exposure to inflammation despite both cohorts having the same duration of Kras activation (Figure 4G; Figure S4G). We then asked whether brief Kras $^{\mathrm{G} 12 \mathrm{D}}$ activation-induced genes associated with persistently accessible memory regions. Indeed, we found that DEGs between prior caerulein and prior saline conditions (with superimposed ras $^{\text {G12D }}$ ) were more likely to be associated with cluster 3 (memory) DARs than any other cluster (Figure 4H). Additionally, we found that the leadingedge ADM transcripts that define the transcriptional component of memory (see Figure 2I; 'ADM primed') were more likely to be differentially expressed between prior caerulein and prior saline mice in the context of brief $\mathrm{Kras}^{\mathrm{G} 12 \mathrm{D}}$ activation (Figure 4I). Unexpectedly, the leading-edge acinar transcripts slightly diminished in samples exposed to prior inflammation (see Figure 2l; 'acinar primed') were not further downregulated upon brief activation of $\mathrm{Kras}^{\mathrm{G12D}}$ (Figure 4J). Among the most differentially expressed genes between prior caerulein $+K_{r a s}{ }^{G 12 D}$ and prior saline $+K_{r a s}{ }^{G 12 D}$ conditions were the PanIN transcripts Pgc, Krt7, Gkn2, and Gkn3, all of which have nearby putative enhancers displaying increased accessibility long after prior inflammation (i.e. cluster 3-type) (Figure $\mathrm{S} 4 \mathrm{H}$ ). These findings suggest that the memory of prior inflammation is selectively recalled in the response to delayed $K_{r a s}{ }^{G 12 D}$ activation, wherein we observe a lowered threshold for tumorigenesis.

To validate these findings, we performed scRNA-seq in mice treated with saline or caerulein followed by $12 \mathrm{w}$ recovery, before and after 2 days of mutant Kras activation. In these experiments, we observed little to no differences in gene expression prior to superimposed Kras (Figure 4J). However, after Kras activation, we saw different emergent cell populations closely related to normal acinar cells. Incipient PanIN cells in the prior caerulein + Kras $^{G 12 D}$ condition were notable for higher expression of Gkn3 and Krt19 and loss of Cpa1; by contrast, prior saline $+K$ ras $^{\mathrm{G12D}}$ featured distinct cells resembling ADM, with high levels of $K r+19$ and S100a6 but no Gkn3 (Figure 4K). This is in line with the mouse histology, which was notable for ADM but no PanIN in the absence of memory of prior inflammation. Together, these single-cell data confirm the presence of a divergent response to mutant Kras between naïve and inflammationresolved mice that appears closely related to the molecular 
A Mist1-Cre ${ }^{\text {ERT2; }}$

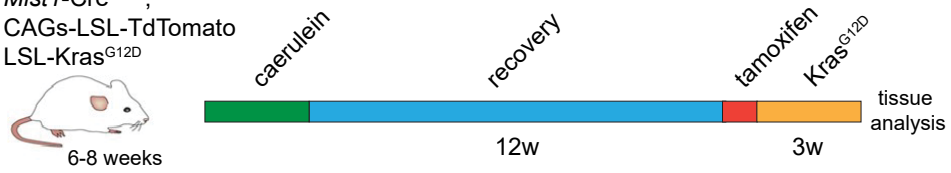

C

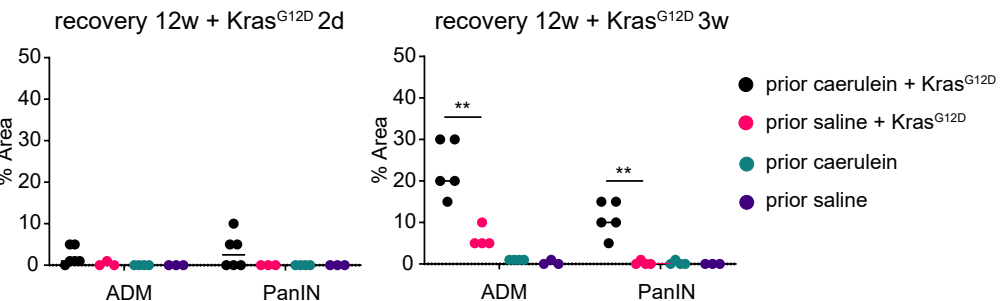

E
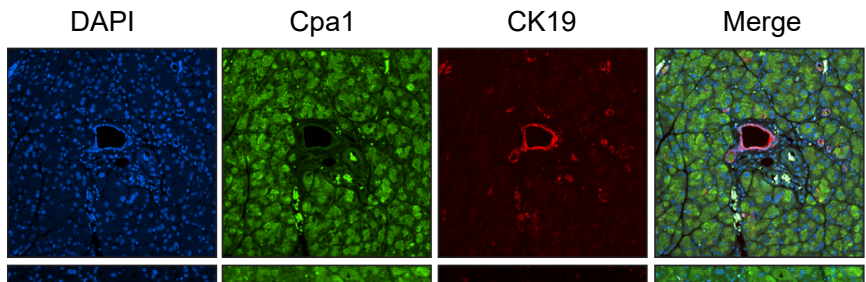

흔
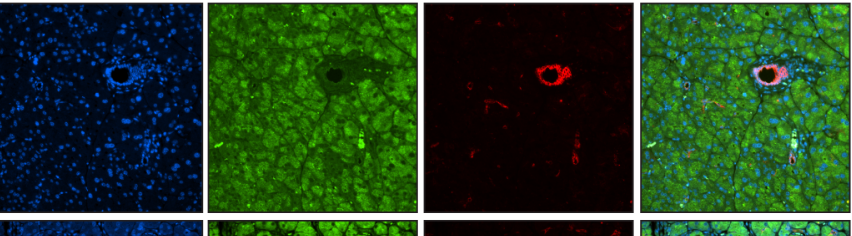

$\frac{.}{0}$
$\frac{1}{3}$
$\frac{2}{0}$
$\frac{0}{0}$
.0
$\frac{0}{0}$
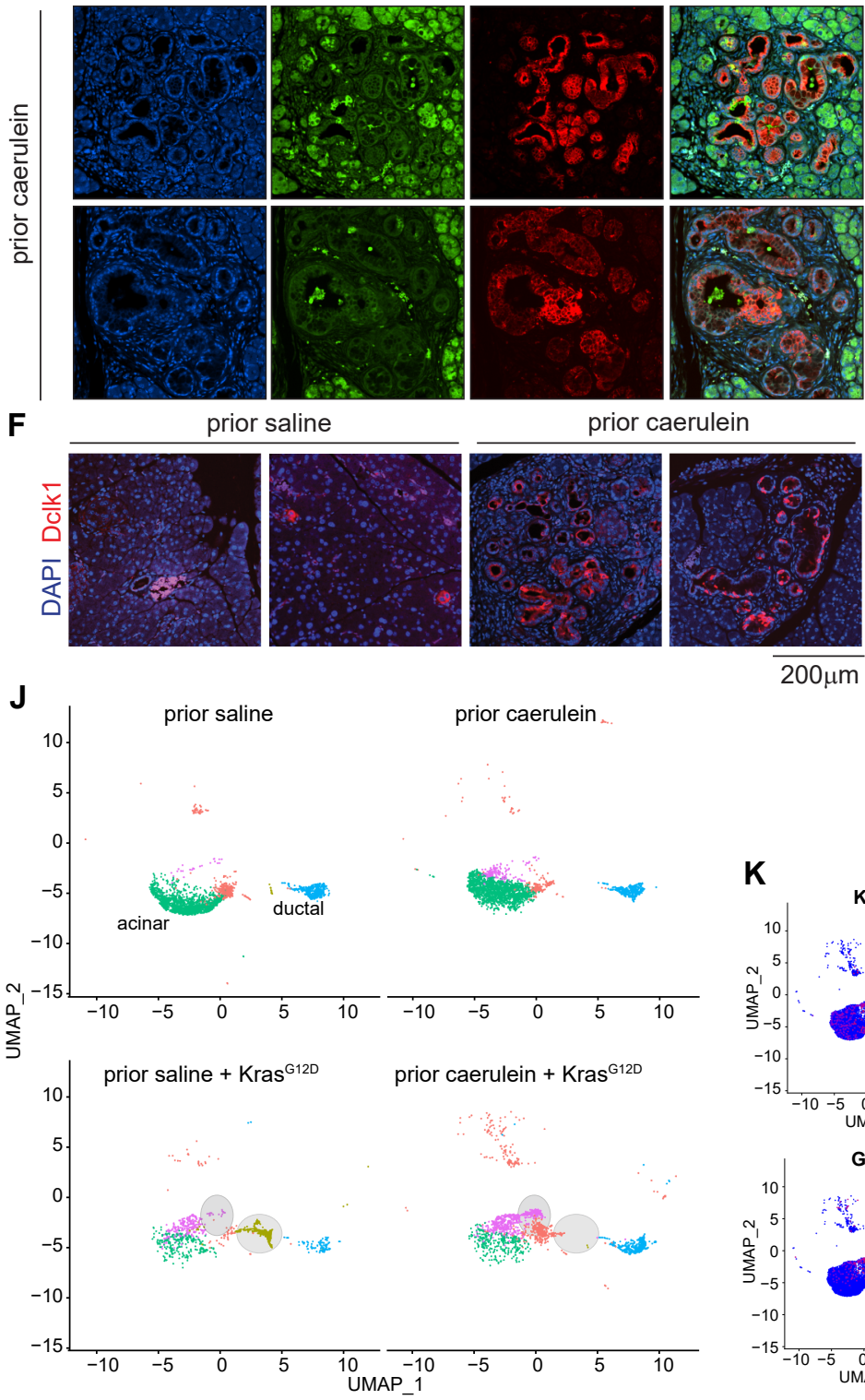

B
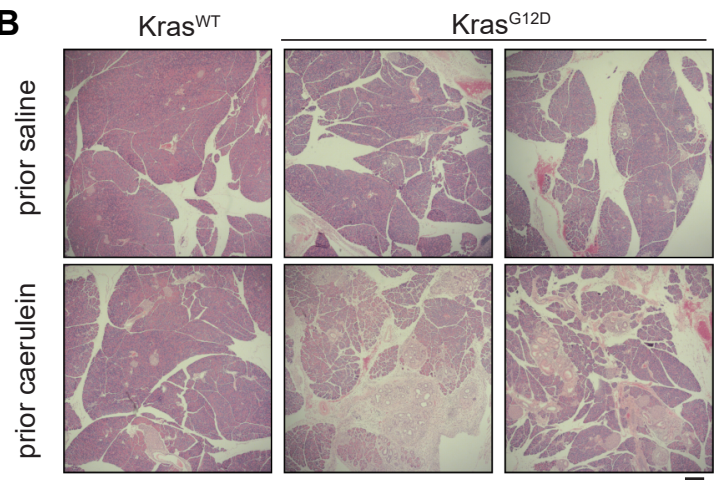

$100 \mu \mathrm{m}$

D

$200 \mu \mathrm{m}$
G

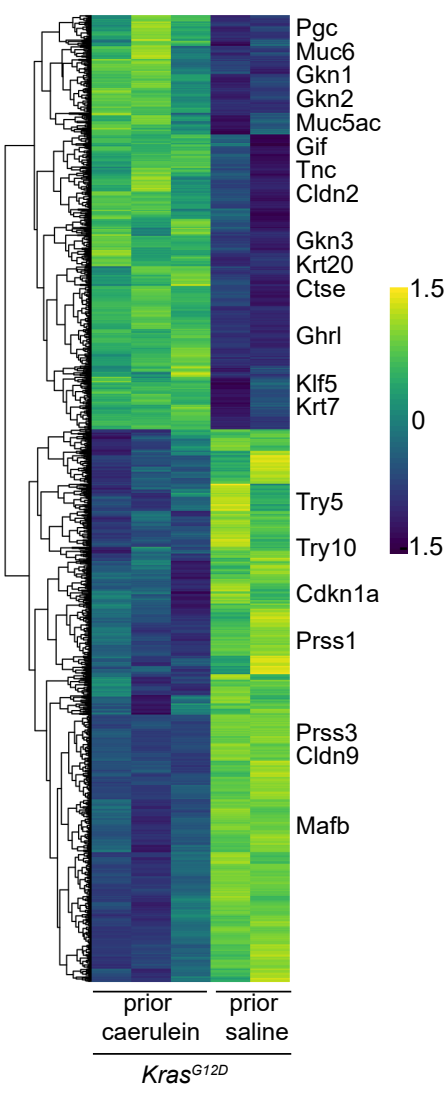

H

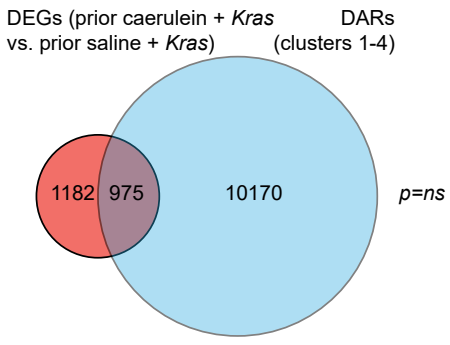

DEGs (prior caerulein + Kras DARs vs. prior saline + Kras) (cluster 3 )
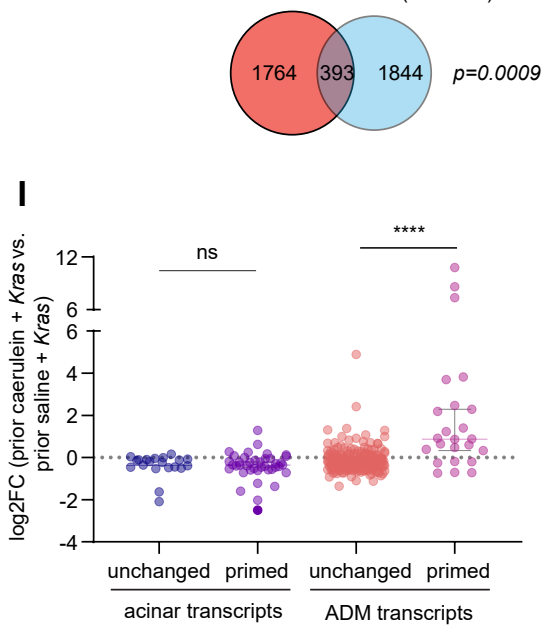

$\mathbf{L}$
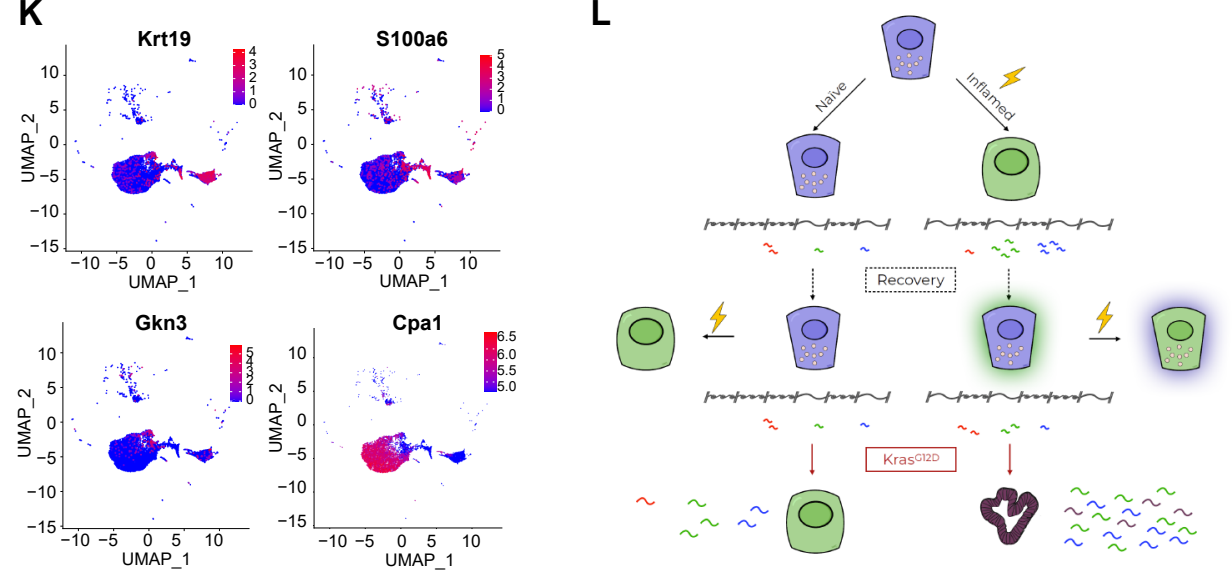
bioRxiv preprint doi: https://doi.org/10.1101/2021.11.01.466807; this version posted November 4, 2021. The copyright holder for this preprint (which was not certified by peer review) is the author/funder. All rights reserved. No reuse allowed without permission.

Falvo et al, Epigenetic memory of inflammation in acinar cells potentiates Kras-driven pancreatic tumorigenesis

alterations immediately preceding oncogene activation.

\section{DISCUSSION}

Inflammation is well-understood to potentiate the development of cancer through both cell-extrinsic and cellintrinsic mechanisms. In the pancreas, the role of macrophages $^{17-18}$, fibroblasts ${ }^{19}$, and $T$ cell subsets ${ }^{15}$ in orchestrating the inflammatory response have been welldescribed. These local cues potentiate a cell-intrinsic alteration in acinar cell fate wherein they acquire duct-like features $^{20}$ and dedifferentiated programs ${ }^{11-12}$. We demonstrate here that the cell-intrinsic effects of inflammation are durable over time, and contribute to cooperativity with oncogenic Kras, even with significant temporal separation between these stressors.

In the absence of mutant Kras, acinar cells theoretically retain the capacity to redifferentiate. Indeed, it has long been demonstrated that there is histologic resolution of inflammation just a few weeks after caerulein. Using our mouse models, we surprisingly observe that a normal microscopic appearance belies ongoing changes to both gene expression and chromatin that continue weeks after supposed resolution of inflammation. Moreover, we demonstrate that 'redifferentiation' is actually an incomplete cell fate decision, wherein ADM chromatin features are partially retained and certain acinar features are lost. This property appears specific to the acinar compartment, whereby we observe neither lasting alterations in other cellular compartments of the microenvironment nor to cellintrinsic expression of typical markers of cell identity in the pancreas. The cell-specificity in our studies allows for us to detect that acinar cell regeneration is incomplete, raising the question of whether physiologic and in vitro forced differentiation and dedifferentiation also features persistence of prior cell identity. ${ }^{21}$

This epigenetic memory of inflammation appears durable over time, and up to 3 months removed from the initial insult, retains context-specific effects on subsequent acinar cell responses. Prior inflammation limits ADM in response to a secondary insult with caerulein with no apparent downregulation of zymogen production or associated tissue damage, suggesting a homeostatic adaptation that does not require metaplasia to resist tissue-level injury. Whereas ADM has long been suggested to serve a protective role, we observe that the long-term effect of inflammation after 12 weeks has the paradoxical effect of circumventing ADM altogether. Whether this feature might be owing to diminished expression of the cholecystokinin receptor at the cell surface or of other intermediate signaling molecules is unclear. These data diverge from recent findings ${ }^{22}$ suggesting that the propensity for ADM is heightened by memory of prior inflammation, although these studies were performed with a 28 day recovery period. Indeed, we also observe that a short recovery ( 21 days) yields an enhanced effect with prior caerulein, which we attribute to residual inflammation and the ongoing molecular dynamics in the first several weeks after inflammation. Prior inflammation thus does not uniformly disrupt epithelial differentiation, but appears to skew responses in a context- and timedependent fashion.

Importantly, the cell-autonomous and in vivo responses to EGFR activation or to mutant Kras signaling are potentiated by prior inflammation. We also show for the first time that there is bona fide 'recall' of both epigenetic and transcriptional components of memory, insofar as the response to delayed Kras is associated with elevated transcription of specific memory-associated genes. Indeed, our single-cell data recapitulate these findings, as $\mathrm{Kras}^{\mathrm{G} 12 \mathrm{D}}$ in the context of prior caerulein unveils a PanIN cell population not present after $\mathrm{Kras}^{\mathrm{G}}{ }^{12 \mathrm{D}}$ is turned on in prior saline mice. Interestingly, in these latter mice, superimposed Kras yields $A D M$, mirroring the findings with caerulein re-challenge (Figure 4L). Putative enhancers and genes related to and downstream of MAPK signaling, itself critical to both metaplastic and regenerative components of the response to inflammation ${ }^{23}$, appear to be specifically enriched in memory and recall.

Thus, our data demonstrate that transcriptional and epigenetic reprogramming in response to inflammation yields persistent alterations to the pre-neoplastic acinar cell; in turn, those precise inflammation-induced alterations are amplified with secondary and substantially delayed oncogenic stress. These data thus demonstrate that longlived differentiated epithelial cells have the capacity to 'remember', and show that the threshold for tumor development can also be durably altered. How this memory is propagated and its specific encoding in chromatin beyond accessibility remain open questions. Closer analyses of TF engagement or histone modifications at key regulatory elements that define memory warrant further study. In

\footnotetext{
Figure 4. Prior inflammation lowers the threshold for subsequent Kras-driven tumor initiation. A, Schematic representation of lineage-traced mouse model with initial exposure to pancreatitis, prolonged recovery, and delayed Kras ${ }^{G 12 D}$ activation. $\mathbf{B}$, Hematoxylin and eosin staining of inflammation-naïve and inflammation-exposed mouse pancreas sections collected after 12 weeks of recovery and 3 weeks of mutant Kras activation. Representative images shown are from a total of $\mathrm{N}=4-5$ mice per condition. $\mathrm{C}$, Histologic quantification of ADM and PanIN lesions in percentage of pancreas sections collected from mice exposed to the corresponding conditions wherein duration of Kras ${ }^{612 D}$ activation is either 2 days or 3 weeks. D, Alcian blue staining of pancreas

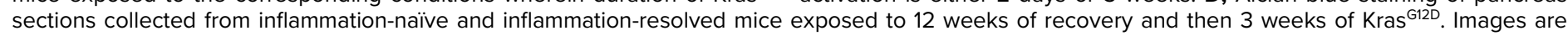
representative of $\mathrm{N}=4-5$ mice per condition. $\mathbf{E} \& \mathbf{F}$, Immunofluorescence for (E) Cpa1 and CK19 and (F) Dclk1 and DAPI staining of pancreas sections collected from inflammation-naïve and inflammation-resolved mice exposed to 12 weeks of recovery and then 3 weeks of mutant Kras. Representative images shown are from a total of $\mathrm{N}=2-5$ mice per condition. G, Heatmap of differentially expressed genes between inflammation-naïve and inflammation-resolved tdTomato(+) acinar cells after 12 weeks of recovery and then 2 days of Kras ${ }^{G 12 D}$. Threshold of $p<0.01$ was used to identify DEGs. Selected genes are highlighted from both cluster. $\mathbf{H}$, Venn diagrams illustrating the degree of overlap between differentially expressed genes (DEGs) altered with prior caerulein plus brief Kras ${ }^{\mathrm{G} 12 \mathrm{D}}$ activation and genes associated with differentially accessible regions (DARs) enriched in respective clusters. Fisher's exact test was used. I, Dot plot illustrating the fold change of leading edge 'acinar transcripts' (primed) and leading edge 'ADM transcripts' (primed) compared to unchanged 'acinar transcripts' and unchanged 'ADM transcripts' (identified from Figure $2 \mathrm{l}-\mathrm{J}$ ) in inflammation-naïve and inflammation-resolved tdTomato(+) acinar cells exposed to 12 weeks of recovery and then 2 days of Kras ${ }^{\mathrm{G} 12 \mathrm{D}}$. Student's t-test was performed to compare conditions. J, UMAP of single-cell RNA-seq data plotting only the epithelial cell compartment (e.g. acinar, ductal) in inflammation-naïve and inflammation-resolved tdTomato(+) acinar cells exposed to 12 weeks of recovery before (upper panels) and after (lower panels) 2 days of Kras ${ }^{612 D}$; gray circles highlight condition-specific cell populations. $\mathbf{K}$, Feature plots generated with single-cell RNA-seq data illustrating the expression of ductal/PanIN and acinar markers.
} 
bioRxiv preprint doi: https://doi.org/10.1101/2021.11.01.466807; this version posted November 4, 2021. The copyright holder for this preprint (which was not certified by peer review) is the author/funder. All rights reserved. No reuse allowed without permission.

Falvo et al, Epigenetic memory of inflammation in acinar cells potentiates Kras-driven pancreatic tumorigenesis

addition, whether epigenetic memory can be reversed - by either interfering with the priming of dysregulated enhancers and associated genes or their recall by secondary stimuli will be a critical question moving forward. Both the MAPK pathway and AP-1 transcription factors could represent intriguing targets in this vein. Finally, how the cell-intrinsic memory affects crosstalk with other cell types remains of importance. We do not observe significant changes to either immune or stromal compartments, but more detailed studies in this arena are certainly warranted. Overall, our findings serve to deepen further the close relationship between inflammation and cancer, such that inducing epigenetic 'amnesia' of an inflammatory insult could be leveraged as a novel cancer prevention strategy.

\section{MATERIALS AND METHODS}

\section{Animal models}

Mice were housed in a pathogen-free facility at Weill Cornell Medicine (WCM). All manipulations were performed under the Institutional Animal Care and Use Committee (IACUC)-approved protocol (2017-0038). Male and female animals were used for experiments. Tamoxifen and caerulein were administered as described previously. ${ }^{15}$ Mice were euthanized and single-cell suspensions were generated (as detailed in Supplemental Methods).

\section{RNA-seq and ATAC-seq}

Sorted acinar-derived DAPI-/tdTomato+ cells were subjected to TRIzol RNA extraction (RNA-seq) or to nuclear isolation (ATAC-seq). Libraries were prepared as described previously ${ }^{10}$ with modifications and analysis as detailed in Supplemental Methods.

\section{Acinar 3D cell culture preparation}

Acinar 3D culture we generated as per Shi et al. ${ }^{24}$ with few modifications. Briefly, acinar cell isolation was obtained with collagenase/dispase mix dissociation as described above, then cells were filtered through a $100 \mu \mathrm{m}$ cell strainer. Pellet was resuspended in AE media composed of DMEM supplemented with $1 \%$ FBS, $1 \%$ PS, $1 \%$ STI and $1 \mathrm{mg} / \mathrm{ml}$ of Dexamethasone and then mixed with Matrigel (Corning) in a 2:1 ratio cells:Matrigel. Per 24well plate, $400 \mu$ of the cells:Matrigel suspension were plated and incubated at $37^{\circ} \mathrm{C}$ for solidification for at least 1 hour. Upon Matrigel solidification, $400 \mu$ l of warm AE media was added with or without TGF- $\alpha$ at $50 \mathrm{ng} / \mathrm{ml}$ concentration. Media (with and without TGF- $\alpha$ ) was changed at day 1 and day 3 of culture. Brightfield image at $4 X$ and 10X magnification were taken using a Nikon ECLIPSE $\mathrm{Ti}$ inverted microscope system equipped with an Andor Zyla 5.5 sCMOS camera. Analysis is as detailed in Supplemental Methods.

\section{Histopathology and Immunofluorescence staining}

Pancreata were fixed overnight in $4 \%$ buffered PFA, transferred to $70 \%$ ethanol, and then embedded in paraffin using IDEXX BioAnalytics laboratory. Serial sections were cut and hematoxylin/eosin staining performed. For IF, slides were deparaffinized, underwent an antigen retrieval using Sodium citrate buffer, blocked with 5\% BSA supplemented with 0.4\% Triton X-100 in PBS, and primary antibodies (Supplementary Table 1) were incubated overnight at $4^{\circ} \mathrm{C}$. Secondary antibodies conjugated with Alexa-488 or Alexa-647 (Invitrogen) were used and DAPI nuclear counterstaining was performed. Fluorescent images were captured with a Nikon ECLIPSE Ti inverted microscope system equipped with an Andor Zyla 5.5 sCMOS camera. For Alcian blue staining, we used the Alcian Blue (ph2.5) Stain Kit (\#H-3501) following the manufacturer's recommendation. H\&E and Alcian blue images were captured with a ZEISS Axio Scope.A1 equipped with a Axiocam 105 color.

\section{Flow cytometry preparation and analysis}

A single cell suspension was generated and red blood cells (RBCs) removed by using the ACK RBC lysis buffer. Cell suspensions were then stained for extracellular markers (after live/dead staining with Zombie Aqua) per the manufacturer's instructions, using primary antibodies conjugated to fluorophores (Supplementary Table 2) in FACS buffer containing 2\% FBS. After washing, cells were resuspended in FACS buffer (2\% FBS), and analyzed on an Attune NxT flow cytometer. Data analyses were performed with FlowJo software.

\section{Single-cell RNA-sequencing}

A single cell suspension was generated and subjected to $10 x$ Chromium scRNA-seq as per the manufacturer's instruction. Analysis was performed using Seurat ${ }^{25}$ as detailed in Supplemental Methods.

\section{ACKNOWLEDGEMENTS}

We thank L. Dow, S. Josefowicz, R. Niec, E. Piskounova, J. Pitarresi, and A. Rustgi for critical readings of the manuscript. This work was supported by an American Association for Cancer ResearchPancreatic Cancer Action Network Pathway to Leadership Award (RC), Emerson Collective Cancer Research Fund (RC), and an American Surgical Association Fellowship Award (to RC). DJF is supported by a NIH/NCI Ruth L. Kirschstein NRSA F31CA265166; $A G$ is supported by the Prevent Cancer Foundation; SLB and AFR are supported by a $\mathrm{NCl}$ T32CA203702 grant.

\section{AUTHOR CONTRIBUTIONS}

DJF designed and performed experiments, analyzed data, and wrote the paper. AG performed experiments, analyzed data, and wrote the paper. AO, SB, and GP performed experiments. PZ, JY, AFR, and FD analyzed data. OE, DB, and RK supervised data analysis. RC designed, performed, and supervised experiments, analyzed data, supervised data analysis, and wrote the paper.

\section{CONFLICTS OF INTEREST}

The authors have no conflicts to disclose.

\section{REFERENCES}

1. Ostuni R, Piccolo V, Barozzi I, Polletti S, Termanini A, Bonifacio S, Curina A, Prosperini E, Ghisletti S, Natoli G. Latent enhancers activated by stimulation in differentiated cells. Cell. 2013 Jan 17;152(1-2):157-71. doi: 10.1016/j.cell.2012.12.018. PMID: 23332752.

2. Naik S, Larsen SB, Gomez NC, Alaverdyan K, Sendoel A, Yuan S, Polak L, Kulukian A, Chai S, Fuchs E. Inflammatory memory sensitizes skin epithelial stem cells to tissue damage. Nature. 2017 Oct 26;550(7677):475-480. doi: 10.1038/nature24271. Epub 2017 Oct 18. Erratum in: Nature. 2018 Aug;560(7716):E2. PMID: 29045388; PMCID: PMC5808576.

3. Ordovas-Montanes J, Dwyer DF, Nyquist SK, Buchheit KM, Vukovic M, Deb C, Wadsworth MH 2nd, Hughes TK, Kazer SW, Yoshimoto E, Cahill KN, Bhattacharyya N, Katz HR, Berger B, Laidlaw TM, Boyce JA, Barrett NA, Shalek AK. Allergic inflammatory memory in human respiratory epithelial progenitor cells. Nature. 2018 Aug;560(7720):649-654. doi: 10.1038/s41586-018-0449-8. Epub 2018 Aug 22. PMID: 30135581; PMCID: PMC6133715.

4. Grimont A, Leach SD, Chandwani R. Uncertain Beginnings: Acinar and Ductal Cell Plasticity in the Development of Pancreatic Cancer. Cell Mol Gastroenterol Hepatol. 2021 Aug 2:S2352345X(21)00160-0. doi: 10.1016/j.jcmgh.2021.07.014. Epub ahead of print. PMID: 34352406.

5. Guerra C, Collado M, Navas C, Schuhmacher AJ, HernándezPorras I, Cañamero M, Rodriguez-Justo M, Serrano M, Barbacid M. Pancreatitis-induced inflammation contributes to pancreatic cancer by inhibiting oncogene-induced senescence. Cancer Cell. 2011 Jun 14;19(6):728-39. doi: 10.1016/j.ccr.2011.05.011. PMID: 21665147; PMCID: PMC4890723.

6. Guerra C, Schuhmacher AJ, Cañamero M, Grippo PJ, 
bioRxiv preprint doi: https://doi.org/10.1101/2021.11.01.466807; this version posted November 4, 2021. The copyright holder for this preprint (which was not certified by peer review) is the author/funder. All rights reserved. No reuse allowed without permission.

Falvo et al, Epigenetic memory of inflammation in acinar cells potentiates Kras-driven pancreatic tumorigenesis

Verdaguer L, Pérez-Gallego L, Dubus P, Sandgren EP, Barbacid M. Chronic pancreatitis is essential for induction of pancreatic ductal adenocarcinoma by K-Ras oncogenes in adult mice. Cancer Cell. 2007 Mar;11(3):291-302. Doi: 10.1016/j.ccr.2007.01.012. PMID: 17349585.

7. Habbe N, Shi G, Meguid RA, Fendrich V, Esni F, Chen $H$, Feldmann G, Stoffers DA, Konieczny SF, Leach SD, Maitra A. Spontaneous induction of murine pancreatic intraepithelial neoplasia (mPanIN) by acinar cell targeting of oncogenic Kras in adult mice. Proc Natl Acad Sci U S A. 2008 Dec 2;105(48):18913-8. 8. Kopp JL, von Figura G, Mayes E, Liu FF, Dubois CL, Morris JP 4th, Pan FC, Akiyama H, Wright CV, Jensen K, Hebrok M, Sander M. Identification of Sox9-dependent acinar-to-ductal reprogramming as the principal mechanism for initiation of pancreatic ductal adenocarcinoma. Cancer Cell. 2012 Dec 11;22(6):737-50.

9. Cobo I, Martinelli P, Flández M, Bakiri L, Zhang M, Carrillo-deSanta-Pau E, Jia J, Sánchez-Arévalo Lobo VJ, Megías D, Felipe I, Del Pozo N, Millán I, Thommesen L, Bruland T, Olson SH, Smith J, Schoonjans K, Bamlet WR, Petersen GM, Malats N, Amundadottir LT, Wagner EF, Real FX. Transcriptional regulation by NR5A2 links differentiation and inflammation in the pancreas. Nature. 2018 Feb 22;554(7693):533-537.

10. Alonso-Curbelo D, Ho YJ, Burdziak C, Maag JLV, Morris JP 4th, Chandwani R, Chen HA, Tsanov KM, Barriga FM, Luan W, Tasdemir N, Livshits G, Azizi E, Chun J, Wilkinson JE, Mazutis L, Leach SD, Koche R, Pe'er D, Lowe SW. A gene-environment-induced epigenetic program initiates tumorigenesis. Nature. 2021 Feb;590(7847):642-648. doi: 10.1038/s41586-020-03147-x. Epub 2021 Feb 3. PMID: 33536616; PMCID: PMC8482641.

11. Means AL, Meszoely IM, Suzuki K, Miyamoto Y, Rustgi AK, Coffey RJ Jr, Wright CV, Stoffers DA, Leach SD. Pancreatic epithelial plasticity mediated by acinar cell transdifferentiation and generation of nestin-positive intermediates. Development. 2005 Aug;132(16):3767-76.

12. Li Y, He Y, Peng J, Su Z, Li Z, Zhang B, Ma J, Zhuo M, Zou D, Liu X, Liu X, Wang W, Huang D, Xu M, Wang J, Deng H, Xue J, Xie W, Lan X, Chen M, Zhao Y, Wu W, David CJ. Mutant Kras co-opts a proto-oncogenic enhancer network in inflammation-induced metaplastic progenitor cells to initiate pancreatic cancer. Nature Cancer volume 2, pages49-65 (2021).

13. Westphalen CB, Takemoto $Y$, Tanaka T, Macchini M, Jiang Z, Renz BW, Chen X, Ormanns S, Nagar K, Tailor Y, May R, Cho Y, Asfaha S, Worthley DL, Hayakawa Y, Urbanska AM, Quante M, Reichert M, Broyde J, Subramaniam PS, Remotti H, Su GH, Rustgi AK, Friedman RA, Honig B, Califano A, Houchen CW, Olive KP, Wang TC. Dclk1 Defines Quiescent Pancreatic Progenitors that Promote Injury-Induced Regeneration and Tumorigenesis. Cell Stem Cell. 2016 Apr 7;18(4):441-55. doi: 10.1016/j.stem.2016.03.016. PMID: 27058937; PMCID: PMC4826481.

14. Kirkegård J, Cronin-Fenton D, Heide-Jørgensen U, Mortensen FV. Acute Pancreatitis and Pancreatic Cancer Risk: A Nationwide Matched-Cohort Study in Denmark. Gastroenterology. 2018 May;154(6):1729-1736.

15. McAllister F, Bailey JM, Alsina J, Nirschl CJ, Sharma R, Fan H, Rattigan Y, Roeser JC, Lankapalli RH, Zhang H, Jaffee EM, Drake CG, Housseau F, Maitra A, Kolls JK, Sears CL, Pardoll DM, Leach SD. Oncogenic Kras activates a hematopoietic-to-epithelial IL-17 signaling axis in preinvasive pancreatic neoplasia. Cancer Cell. 2014 May 12;25(5):621-37. doi: 10.1016/j.ccr.2014.03.014. PMID: 24823639; PMCID: PMC4072043.

16. Schlesinger $Y$, Yosefov-Levi O, Kolodkin-Gal D, Granit RZ, Peters L, Kalifa R, Xia L, Nasereddin A, Shiff I, Amran O, Nevo Y, Elgavish S, Atlan K, Zamir G, Parnas O. Single-cell transcriptomes of pancreatic preinvasive lesions and cancer reveal acinar metaplastic cells' heterogeneity. Nat Commun. 2020 Sep 9;11(1):4516. doi: 10.1038/s41467-020-18207-z. PMID: 32908137; PMCID: PMC7481797.

17. Liou GY, Döppler H, Necela B, Krishna M, Crawford HC, Raimondo M, Storz P. Macrophage-secreted cytokines drive pancreatic acinar-to-ductal metaplasia through NF-KB and MMPs. J Cell Biol. 2013 Aug 5;202(3):563-77. doi: 10.1083/jcb.201301001. PMID: 23918941; PMCID: PMC3734091.

18. Zhang Y, Yan W, Mathew E, Kane KT, Brannon A 3rd, Adoumie M, Vinta A, Crawford HC, Pasca di Magliano M. Epithelial-Myeloid cell crosstalk regulates acinar cell plasticity and pancreatic remodeling in mice. Elife. 2017 Oct 5;6:e27388. doi: 10.7554/eLife.27388. PMID: 28980940; PMCID: PMC5690281.

19. Liu X, Pitarresi JR, Cuitiño MC, Kladney RD, Woelke SA, Sizemore GM, Nayak SG, Egriboz O, Schweickert PG, Yu L, Trela S, Schilling DJ, Halloran SK, Li M, Dutta S, Fernandez SA, Rosol TJ, Lesinski GB, Shakya R, Ludwig T, Konieczny SF, Leone G, Wu J, Ostrowski MC. Genetic ablation of Smoothened in pancreatic fibroblasts increases acinar-ductal metaplasia. Genes Dev. 2016 Sep 1;30(17):1943-55. doi: 10.1101/gad.283499.116. Epub 2016 Sep 15. PMID: 27633013; PMCID: PMC5066238.

20. Reichert M, Takano S, von Burstin J, Kim SB, Lee JS, IhidaStansbury K, Hahn C, Heeg S, Schneider G, Rhim AD, Stanger BZ, Rustgi AK. The Prrx1 homeodomain transcription factor plays a central role in pancreatic regeneration and carcinogenesis. Genes Dev. 2013 Feb 1;27(3):288-300. doi: 10.1101/gad.204453.112. Epub 2013 Jan 25. PMID: 23355395; PMCID: PMC3576514.

21. Jadhav U, Cavazza A, Banerjee KK, Xie H, O'Neill NK, SaenzVash V, Herbert Z, Madha S, Orkin SH, Zhai H, Shivdasani RA. Extensive Recovery of Embryonic Enhancer and Gene Memory Stored in Hypomethylated Enhancer DNA. Mol Cell. 2019 May 2;74(3):542-554.e5. doi: 10.1016/j.molcel.2019.02.024. Epub 2019 Mar 21. PMID: 30905509; PMCID: PMC6499659.

22. Del Poggetto E, Ho IL, Balestrieri C, Yen EY, Zhang S, Citron F, Shah R, Corti D, Diaferia GR, Li CY, Loponte S, Carbone F, Hayakawa Y, Valenti G, Jiang S, Sapio L, Jiang H, Dey P, Gao S, Deem AK, Rose-John S, Yao W, Ying H, Rhim AD, Genovese G, Heffernan TP, Maitra A, Wang TC, Wang L, Draetta GF, Carugo A, Natoli $G$, Viale A. Epithelial memory of inflammation limits tissue damage while promoting pancreatic tumorigenesis. Science. 2021 Sep 17;373(6561):eabj0486. doi: 10.1126/science.abj0486. Epub 2021 Sep 17. PMID: 34529467.

23. Halbrook CJ, Wen HJ, Ruggeri JM, Takeuchi KK, Zhang Y, di Magliano MP, Crawford HC. Mitogen-activated Protein Kinase Kinase Activity Maintains Acinar-to-Ductal Metaplasia and Is Required for Organ Regeneration in Pancreatitis. Cell Mol Gastroenterol Hepatol. 2017 Jan;3(1):99-118. doi: 10.1016/j.jcmgh.2016.09.009. PMID: 28090569; PMCID: PMC5235341.

24. Shi G, DiRenzo D, Qu C, Barney D, Miley D, Konieczny SF. Maintenance of acinar cell organization is critical to preventing Kras-induced acinar-ductal metaplasia. Oncogene. 2013 Apr 11;32(15):1950-8. doi: 10.1038/onc.2012.210. Epub 2012 Jun 4. PMID: 22665051; PMCID: PMC3435479.

25. Stuart T, Butler A, Hoffman P, Hafemeister C, Papalexi E, Mauck WM 3rd, Hao Y, Stoeckius M, Smibert P, Satija R. Comprehensive Integration of Single-Cell Data. Cell. 2019 Jun 13;177(7):18881902.e21. doi: 10.1016/j.cell.2019.05.031. Epub 2019 Jun 6. PMID: 31178118; PMCID: PMC6687398. 